\title{
Importance of Preserving Parathyroids in Surgery for Hemiagenesis of Thyroid: Analysis with Review of Literature
}

\author{
${ }^{1}$ Sudhir M Naik, ${ }^{2}$ Rajshekar Halkud, ${ }^{3}$ Purshottam Chavan, ${ }^{4}$ Mohan Appaji, ${ }^{5} \mathrm{P}$ Sathya, ${ }^{6}$ S Ravishankar Bhat, ${ }^{7}$ RM Deekshith
}

\begin{abstract}
Background/objectives: As congenital anomalies of the thyroid are rare and the pathology associated with it warranting total thyroidectomies is still rare, so the inadvertent parathyroidectomies are rarely reported. Unilateral or bilateral hypoplasia or agenesis of one or both thyroid lobes, with or without isthmic agenesis, is a rare developmental anomaly. Meticulous subcapsular dissection with preserving the vascular twigs to the parathyroid is very important in avoiding complications.
\end{abstract}

Materials and methods: We report two cases of thyroid hemiagenesis in our medical college institution over a period of 10 years. Both were females in the 6th decade with swelling in the neck. One had a right-sided swelling and the second had a left-sided swelling, both measuring about $5 \times 5 \mathrm{~cm}$.

Conclusion: So the total thyroidectomy surgery in hemiagenesis cases poses a risk to the parathyroids as the altered anatomy. So the time-tested technique of subcapsular dissection and meticulous preservation of the multiple vasculature of the parathyroids in the fascial compartment provides better results with least morbidity.

Keywords: Hemiagenesis, Inferior parathyroid artery, Parathyroid, Recurrent laryngeal nerve.

How to cite this article: Naik SM, Halkud R, Chavan P, Appaji M, Sathya P, Bhat SR, Deekshith RM. Importance of Preserving Parathyroids in Surgery for Hemiagenesis of Thyroid: Analysis with Review of Literature. Int J Head Neck Surg 2015;6(4):146-148.

Source of support: Nil

Conflict of interest: None

\section{INTRODUCTION}

As congenital anomalies of the thyroid are rare and the pathology associated with it warranting total thyroidectomies is still rare, so the inadvertent parathyroidectomies

${ }^{1}$ Professor and Head, ${ }^{2,4}$ Professor, ${ }^{3,6}$ Associate Professor ${ }^{5}$ Professor and Medical Superintendant, ${ }^{7}$ Assistant Professor

${ }^{1,4-7}$ Department of ENT and Head and Neck Surgery, KVG Medical College and Hospital Sullia, Karnataka, India

${ }^{2,3}$ Department of Head and Neck Oncosurgery, Kidwai Memorial Institute of Oncology, Bengaluru, Karnataka, India

Corresponding Author: Sudhir M Naik, Professor and Head, Department of ENT and Head and Neck Surgery, KVG Medical College and Hospital Sullia, Karnataka, India, Phone: +919916807109, e-mail: sud223@gmail.com are rarely reported. Unilateral or bilateral hypoplasia or agenesis of one or both thyroid lobes, with or without isthmic agenesis, is a rare developmental anomaly. ${ }^{1,2}$ Hemiagenesis of the left lobe is far commoner than of the right. ${ }^{1,2}$ Hemiagenesis of either lobe, with or without agenesis of the isthmus, is very rare, with a prevalence rate of around $0.2 \%$ in asymptomatic children. ${ }^{1,2}$ The left side is the most commonly involved in hemiagenesis $(80 \%))^{3-5}$ Clinically, these patients may be euthyroid, hyperthyroid, or hypothyroid..$^{3-5}$ The isthmus may be absent in $40.50 \%$ of cases. ${ }^{3-5}$ Thyroid function may be altered in $38.47 \%$ of patients. ${ }^{3-5}$ Recognition of this rare anomaly by means of ultrasonography is valuable for a complete thyroidectomy and for avoiding unnecessary contralateral dissection and damage. Meticulous subcapsular dissection with preserving the vascular twigs to the parathyroid is very important in avoiding complications.

\section{MATERIALS AND METHODS}

We report two cases of thyroid hemiagenesis in our medical college institution over a period of 10 years. Both were females in the 6th decade with swelling in the neck. One had a right sided swelling and the other a left sided swelling both measuring about $5 \times 5 \mathrm{~cm}$ on palpation. The swelling moved with the protrusion of the tongue and on neck sonography revealed absent right lobe and isthmus in one and the absent left lobe in the other. Both the anomalies were confirmed on contrast-enhanced computed tomography (CT) scans. Later, fine needle aspiration reported papillary carcinoma in the left lobe and colloid nodular goiter in the right lobe of the other. Thyroid profile done reported both the thyroids to be euthyroid. The papillary carcimona in the left lobe was graded as T3NOM0, and the other in the right lobe was to be treated for cosmetic reasons. Total thyroidectomy with central compartment clearance was planned for papillary carcinoma and hemithyroidectomy for the right lobe (Figs 1 and 2). Intraoperative findings were as expected, and greater efforts at preserving the parathyroids were taken with central compartmental clearance. The nodes in the contralateral part of the central compartment was dissected meticulously under 3.5× magnification preserving all arterial twigs to the parathyroids suspecting their hemiagenesis too. Mild drop 


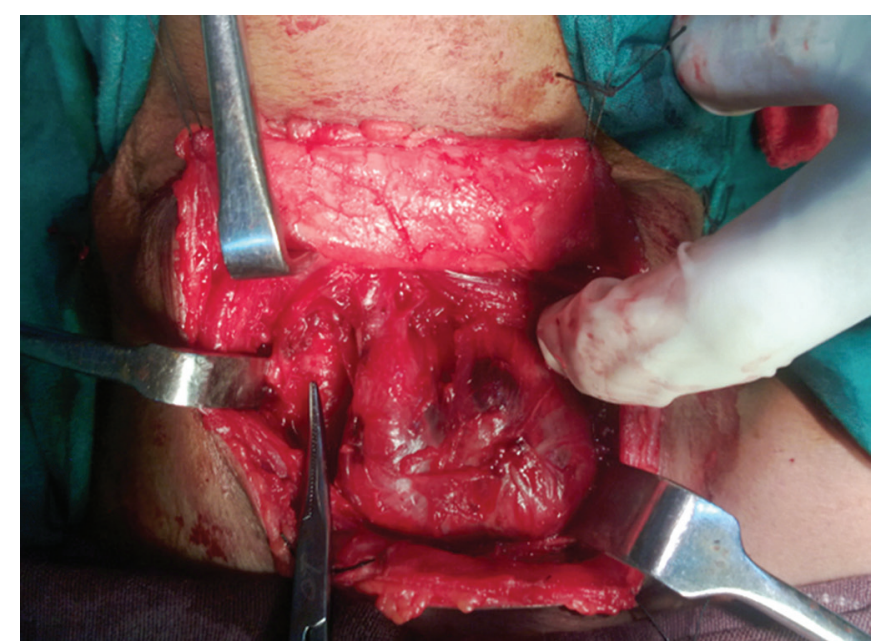

Fig. 1: Absent right lobe of the thyroid

in serum calcium levels due to transient hypocalcemia was seen in the first 3 days, which subsided thereafter. The agenetic lobes were confirmed on gross examination with histopathology showing papillary carcinoma in the left lobe and multinodular goiter in the right lobe.

\section{DISCUSSION}

Congenital thyroid anomalies are rare. ${ }^{6}$ They may be related to abnormal descent of the thyroid gland or to structural abnormalities in thyroid development, such as hypoplasia or hemiagenesis with or without isthmic agenesis. ${ }^{6}$ Left-sided hemiagenesis is far commoner than right-sided hemiagenesis, with a left:right ratio of 4:1.,8 Prevalence is $0.2 \%$. Isthmus is absent in 40 to $50 \%$ cases of hemiagenesis. ${ }^{7,8}$ Most of the cases are sporadic. ${ }^{9}$ Most patients with hemiagenesis are diagnosed incidentally. ${ }^{9}$ Ultrasonography (USG) is a useful modality to detect this anomaly. ${ }^{9}$ In endemic zones, there may be a high incidence of adenomatous nodules and colloid cysts in the single lobe. ${ }^{9}$ Ultrasonography is valuable for a complete thyroidectomy and for avoiding unnecessary contralateral dissection and damage. ${ }^{9}$

We have stressed the importance of parathyroid preservation in all thyroid surgeries with hemiagenesis. Overall, the complications of thyroid surgeries, i.e., recurrent laryngeal nerve injuries, have reduced, and injury or unintentional removal of the parathyroid glands with resultant hypoparathyroidism is more competitive. ${ }^{10}$ The nerve injuries have reduced below single digits even in the hands of beginners and are hardly fatal. And so the surgery of the thyroid is basically to preserve the parathyroid, and hence the emphasis is doubled in case of surgery done for pathology of thyroid hemiagenesis. The parathyroid dysfunction may be evident in 24 to 48 hours depending on the reserves of calcium nutrition of the individual. ${ }^{10}$ The parathyroids vary in their anatomy ranging from 1 to 12 glands in number, but most

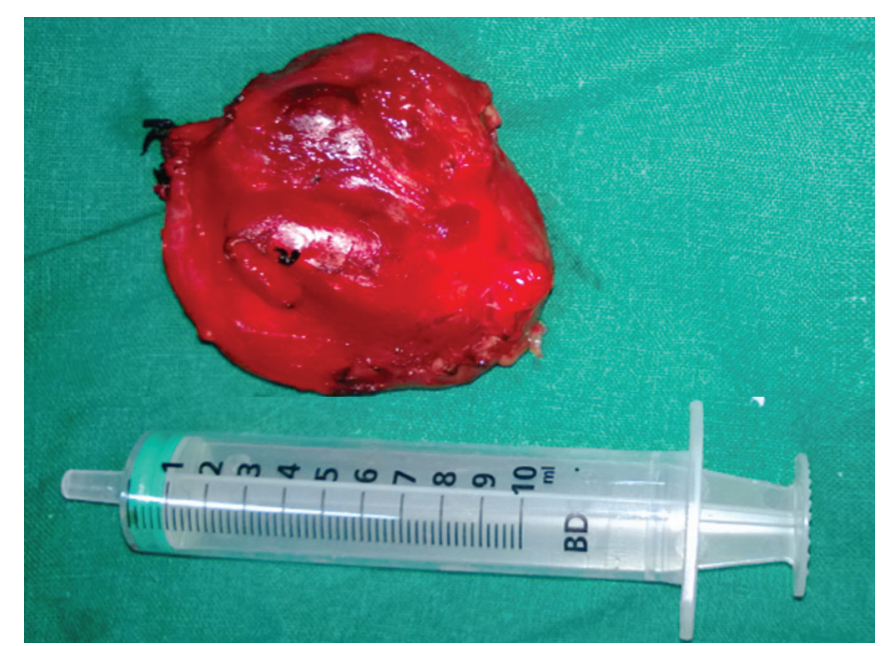

Fig. 2: Hemiagenetic total thyroidectomy specimen

commonly, they are four (two superior and two inferior) in $87 \%$ cases while three in $6 \%$, five in $0.2 \%$, and six in $0.6 \%$ cases. $^{11}$ Intraoperatively, all the four glands can be visualized in total thyroidectomy in only half of the cases, while the area lodging the parathyroids would be the middle $i / 3$ of the thyroid gland posterior surface in two-third of the cases. ${ }^{11}$ The parathyroids weigh $35 \mathrm{mg}$, and the superior glands are consistent in location as they develop from the 4 th branchial arch, which is the same as the thyroid. The superior are located within $1 \mathrm{~cm}$ of the cricoarytenoid joint on the posterior surface of the upper pole of the thyroid gland. Inferior parathyroids are very variable in their location developing from the 3rd branchial arch, same as the thymus, and can be dragged down for a variable distance in the superior mediastinum along the esophagus. ${ }^{11}$

The surgical planning should be based on the variable nature of the parathyroids and the thorough anatomy of the dysgenesis or hemiagenesis present in the thyroid. Superior parathyroid glands hardly vary in location, with approximately $75 \%$ being located either cricothyroidally or juxtathyroidally, and the remainder are located primarily behind the upper pole of the thyroid gland with a percent variability at being either retro-esophageal or retropharyngeal. Inferior parathyroids show lot of variability $(40 \%)$ at lower pole of the thyroid (anterior or posterior), $40 \%$ in tongue of thymic tissue between the inferior border of the thyroid gland and the clavicle, $15 \%$ juxtathyroidally, $1 \%$ mediastinum, and $2 \%$ ectopic along the migrational path from the base of tongue to the lower neck. ${ }^{12}$ A rare intrathyroid location is seen in 2 to $5 \%$ of the cases, but the main blood supply of both the superior and inferior parathyroids is the inferior thyroid artery with a well-formed fascial compartment. Surgical and functional anatomy is well preserved if the fascial sheath is respected as most of the inferior thyroid artery supplies the parathyroid while very less to the thyroid. ${ }^{12}$ 
Preserving vascularity in surgery for pathology for thyroid hemiagenesis explains the need to ligate the superior thyroid artery after identifying and saving its anastomotic vessel to inferior thyroid artery and ligate the branches of inferior thyroid artery close to the gland. This saves the dissection in the vascular plane and protects not only the external branch of the superior laryngeal nerve and the recurrent laryngeal nerve with all its branches and but also, more importantly, the vascularity of the parathyroid glands. ${ }^{12}$ Also, subcapsular medial and lateral dissection helps a surgeon to be in the avascular plane reducing injuries to the parathyroids in meticulous total thyroidectomies for special cases like hemiagenesis. A cephalocaudal dissection is usually practised with the upper pole of the thyroid with ligating the superior thyroid artery. The posterior branch of the superior thyroid artery, which communicates with the ascending branch of the inferior thyroid artery supplying the superior parathyroid, is separated and saved. ${ }^{12}$

The vascularity of the superior glands are fragile, and they change color very soon and should be dissected in subcapsular plane very close on the posterior surface of the upper thyroid pole as a deep yellow ovoid mass. ${ }^{12}$ Tracing the recurrent laryngeal nerve at its entrance over the cricothyroid joint, dissection is done medially and inferiorly and twigs of the inferior thyroid artery are identified. ${ }^{12}$ Later dissection proceeds inferiorly and laterally, staying in front of and anterior to the inferior thyroid artery ligating the twigs entering the gland. The inferior parathyroids were dissected laterally from the lower pole subcapsularlly keeping the vascular twigs to the gland intact. ${ }^{12}$

In Sorgato et al's study, inadvertent parathyroidectomy was more reported with intraoperative identification of glands and vascular compromise. Completion thyroidectomy results in more parathyroid damage as the operative field is scarred, inflamed, and ecchymosed due to earlier surgery. Central compartment clearance posed more threat as compared to completion thyroidectomy or hemiagenesis surgeries as the lymph nodes camouflage the identity of the parathyroids. ${ }^{13}$ Sasson et al and Lin et al reported more parathyroid damage with neck dissections with thyroidectomy as well gastric pullup as the tracheoesophageal groove lymph nodes also contain some parathyroid tissues. ${ }^{14-16}$

\section{CONCLUSION}

Total thyroidectomy surgery in hemiagenesis cases poses a risk to the parathyroids as the altered anatomy. So the time-tested technique of subcapsular dissection and meticulous preservation of the multiple vasculature of the parathyroids in the fascial compartment provides better results with least morbidity.

\section{REFERENCES}

1. Aslaner A, Aydin M, Ozdere A. Multinodular goiter with thyroid hemiagenesis: a case report and review of the literature. Acta Chir Belg 2005 Sep-Oct;105(5):528-530.

2. Karabay N, Comlekci A, Canda MS, Bayraktar F, Degirmenci B. Thyroid hemiagenesis with multinodular goiter: a case report and review of the literature. Endocr J 2003 Aug;50(4):409-413.

3. Hydovitz JD, Winzelberg GA. Use of ultrasonography to eliminate hemiagenesis of a thyroid lobe in the differential diagnosis of a toxic adenoma (Plummer's disease). Clin Nucl Med 1988 Feb;3(2):144-145.

4. Leckie RG, Delaplain CB, Watabe JT, Darrow M. Thyroid hemiagenesis by I-123 thyroid scan with high-resolution real-time ultrasound confirmation. Clin Nucl Med 1993 Feb;18(2):161-163.

5. De Remigis $P, D^{\prime}$ Angelo M, Bonaduce S, Di Giandomenico V, Sensi S. Comparison of ultrasonic scanning and scintiscanning in the evaluation of thyroid hemiagenesis. J Clin Ultrasound 1985 Oct;13(8):561-563.

6. Veliz J, Pineda G. Thyroid hemiagenesis associated with Basedow-Graves disease: Report of a case. Rev Med Chil 2000 Aug;128(8):896-898.

7. Lee IT, Sheu WH, Lin SY. Familial form of thyroid dysgenesis: Report of thyroid hemiagenesis with accompanying Graves' disease in a woman whose daughter has thyroid agenesis. Horm Res 2003;59(1):47-49.

8. Sakurai K, Amano S, Enomoto K, Matsuo S, Kitajima A. Primary hyperparathyroidism with thyroid hemiagenesis. Asian J Surg 2007 Apr;30(2):151-153.

9. Tiwari PK, Baxi M, Baxi J, Koirala D. Right-sided hemiagenesis of the thyroid lobe and isthmus: a case report. Indian J Radiol Imaging 2008 Nov;18(4):313-315.

10. Mangalgiri AS, Mahore D, Kapre M. Study of a unique 'inverted U' shaped thyroid gland and its clinical importance. Indian J Otolaryngol Head Neck Surg 2014 Apr-Jun;66(2):224-225.

11. KremplGA,Medina JE. Current issues in hyperparathyroidism. Otolaryngol Clin N Am 2003;36:217-228.

12. Abboud B, Sleilaty G, Braidy C, Zeineddine S, Ghorra C, Abadjian G, Tabchy B. Careful examination of thyroid specimen intraoperatively to reduce incidence of inadvertent parathyroidectomy during thyroid surgery. Arch Otolaryngol Head Neck Surg 2007 Nov;133(11):1105-1110.

13. Sorgato N, Pennelli G, Boschin IM, Ide EC, Pagetta C, Piotto A, Toniato A, De Salvo GL, Hindié E, Al-Nahhas A, et al. Can we avoid inadvertent parathyroidectomy during thyroid surgery? In Vivo 2009 May-Jun;23(3):433-439.

14. SassonAR,PingpankJF, Wetherington RW,HanlonAL, RidgeJA. Incidental parathyroidectomy during thyroid surgery does not cause transient symptomatic hypocalcemia. Arch Otolaryngol Head Neck Surg 2001 Mar;127(3):304-308.

15. Lee NJ, Blakey JD, Bhuta S, Calcaterra TC. Unintentional parathyroidectomy during thyroidectomy. Laryngoscope 1999 Aug;109(8):1238-1240.

16. Lin DT, Patel SG, Shaha AR, Singh B, Shah JP. Incidence of inadvertent parathyroid removal during thyroidectomy. Laryngoscope 2002 Apr;112(4):608-611. 